\title{
DARK ENERGY: THE ABSOLUTE ELECTRIC POTENTIAL OF THE UNIVERSE*
}

\author{
JOSE BELTRÁN JIMÉNEZ* and ANTONIO L. MAROTO ${ }^{\dagger}$ \\ Departamento de Física Teórica, \\ Universidad Complutense de Madrid, \\ 28040 Madrid, Spain \\ *jobeltra@fis.ucm.es \\ †maroto@fis.ucm.es
}

Communicated by D. V. Ahluwalia

\begin{abstract}
Is there an absolute cosmic electric potential? The recent discovery of the accelerated expansion of the universe could be indicating that this is certainly the case. In this essay we show that the consistency of the covariant and gauge-invariant theory of electromagnetism is truly questionable when considered on cosmological scales. Out of the four components of the electromagnetic field, Maxwell's theory contains only two physical degrees of freedom. However, in the presence of gravity, one of the "unphysical" states cannot be consistently eliminated, thus becoming real. This third polarization state is completely decoupled from charged matter, but can be excited gravitationally, thus breaking gauge invariance. On large scales the new state can be seen as a homogeneous cosmic electric potential, whose energy density behaves as a cosmological constant.
\end{abstract}

The recent discovery of the accelerated expansion of the universe, and the difficulties found in the context of general relativity (GR) and the standard model (SM) of elementary particles to properly account for this effect, have led to consideration of the possibility that physics on large scales could differ from our well-known small scale laws.

In this context, models in which the description of the own gravitational interaction is modified on large scales with respect to GR have been extensively considered in recent years. Here we will concentrate on the other long-range interaction of nature, and explore the possibility that our standard theory of (quantum) electromagnetism, being valid on small scales, could give rise to unexpected effects on cosmological scales. As a matter of fact, this possibility is perfectly compatible with current experimental limits which have tested electromagnetism only for wavelengths roughly below the solar system scales (1.3 A.U. ${ }^{1}$ ).

* This essay received an honorable mention in the 2009 Essay Competition of the Gravity Research Foundation. It was refereed, not as a regular IJMPD research paper, but as an essay. 
In this essay we will discuss one of the most striking consequences of electromagnetism in the cosmological context, which is the possibility that the universe at large scales not only sets a privileged reference frame, but could also determine an absolute electric potential. Indeed, it is well known that the presence of matter and radiation in the universe implies that, on large scales, the universe as a whole has associated a privileged reference frame. That frame is nothing but the cosmic center-of-mass frame $e^{2,3}$ of the different components (baryonic and dark matter, radiation and dark energy). In the case in which all such components are at rest with respect to each other, the frame can be identified with that of the observers who see an isotropic cosmic microwave background. Thus we can say that, although Lorentz symmetry is locally a good symmetry of space-time, it is broken on large scales by the matter/energy content of the universe. But what about the rest of the gauge symmetries and, in particular, that of electromagnetism? Is it also possible that, although on small scales we see electromagnetic gauge symmetry as an exact symmetry of nature, the actual situation is that it is broken by the content of the universe on large scales? Does it make sense to talk about a privileged electromagnetic gauge? We will argue that dark energy, responsible for the accelerated expansion of the universe, could be nothing but the energy density associated with such absolute electric potential. ${ }^{4,5}$

Let us start by briefly reviewing the standard covariant electromagnetic quantization in Minkowski space-time. ${ }^{6,7}$ The starting point is the action

$$
S=\int d^{4} x\left(-\frac{1}{4} F_{\mu \nu} F^{\mu \nu}+\frac{\xi}{2}\left(\partial_{\mu} A^{\mu}\right)^{2}+A_{\mu} J^{\mu}\right),
$$

which is not invariant under general gauge transformations, but only under residual ones, given by $A_{\mu} \rightarrow A_{\mu}+\partial_{\mu} \theta$, with $\square \theta=0$. The equations of motion obtained from this action read

$$
\partial_{\nu} F^{\mu \nu}+\xi \partial^{\mu}\left(\partial_{\nu} A^{\nu}\right)=J^{\mu}
$$

In order to recover the ordinary Maxwell equation, the Lorenz condition $\partial_{\mu} A^{\mu}=0$ must be imposed so that the $\xi$ term disappears. At the classical level this can be achieved by means of appropriate boundary conditions on the field. Indeed, taking the four-divergence of the above equation, we find that

$$
\square\left(\partial_{\nu} A^{\nu}\right)=0,
$$

where we have made use of current conservation. This means that the field $\partial_{\nu} A^{\nu}$ evolves as a free scalar field, so that if it vanishes for large $|t|$ it will vanish for all time. At the quantum level, the Lorenz condition cannot be imposed as an operator identity, but only in the weak sense, $\partial_{\nu} A^{\nu(+)}|\phi\rangle=0$, where $(+)$ denotes the positive frequency part of the operator and $|\phi\rangle$ is a physical state. This condition is equivalent to requiring that the physical states contain the same number of temporal and longitudinal photons, so that their energy densities, having opposite signs, cancel each other. Thus we see that the Lorenz condition seems to be essential 
in order to recover standard Maxwell equations and get rid of the negative energy states.

Now we move to an expanding universe. The curved space-time version of the action (1) reads

$$
S=\int d^{4} x \sqrt{g}\left(-\frac{1}{4} F_{\mu \nu} F^{\mu \nu}+\frac{\xi}{2}\left(\nabla_{\mu} A^{\mu}\right)^{2}+A_{\mu} J^{\mu}\right)
$$

and the modified Maxwell equations are

$$
\nabla_{\nu} F^{\mu \nu}+\xi \nabla^{\mu}\left(\nabla_{\nu} A^{\nu}\right)=J^{\mu}
$$

Taking again the four-divergence, we get

$$
\square\left(\nabla_{\nu} A^{\nu}\right)=0 .
$$

We see that once again $\nabla_{\nu} A^{\nu}$ behaves as a scalar field which is decoupled from the conserved electromagnetic currents, but it is nonconformally coupled to gravity. This means that, unlike the flat space-time case, this field can be excited from quantum vacuum fluctuations by the expanding background in a completely analogous way to the inflaton fluctuations during inflation. Thus this poses the question of the validity of the Lorenz condition at all times.

In order to illustrate this effect, we will present a toy example. Let us consider quantization in the absence of currents, in a spatially flat expanding background, whose metric is written in conformal time as

$$
d s^{2}=a(\eta)^{2}\left(d \eta^{2}-d \mathbf{x}^{2}\right) .
$$

For the scale factor we assume the following form: $a(\eta)=2+\tanh \left(\eta / \eta_{0}\right)$, where $\eta_{0}$ is constant. This metric is asympotically flat in the remote past and far future. Let us prepare our system in an initial state $|\phi\rangle$ belonging to the physical Hilbert space, i.e. satisfying $\partial_{\nu} \mathcal{A}_{\text {in }}^{\nu(+)}|\phi\rangle=0$ in the initial flat region. We solve the coupled system of equations (5) for the corresponding Fourier modes $\mathcal{A}_{\mu \mathbf{k}}$. Because of the expansion of the universe, the positive frequency modes in the in region with a given temporal or longitudinal polarization $\lambda$ will become a linear superposition of positive and negative frequency modes in the out region and with different polarizations $\lambda^{\prime} .8$

Thus the system will end up in a final state which no longer satisfies the weak Lorenz condition, i.e. in the out region $\partial_{\nu} \mathcal{A}_{\text {out }}^{\nu(+)}|\phi\rangle \neq 0$. This is shown in Fig. 1, where we have computed the final number of temporal and longitudinal photons starting from an initial vacuum state with $n_{0}^{\text {in }}(k)=n_{\|}^{\text {in }}(k)=0$. We see that in the final region $n_{0}^{\text {out }}(k) \neq n_{\|}^{\text {out }}(k)$. Notice that the failure comes essentially from large scales $\left(k \eta_{0} \ll 1\right)$, since on small scales $\left(k \eta_{0} \gg 1\right)$ the Lorenz condition can be restored. Motivated by this fact, in the following we will explore the possibility of quantizing electromagnetism in an expanding universe without imposing this condition.

Let us then assume that the fundamental theory for electromagnetism is given by (4) and is not invariant under general gauge transformations, but only under 


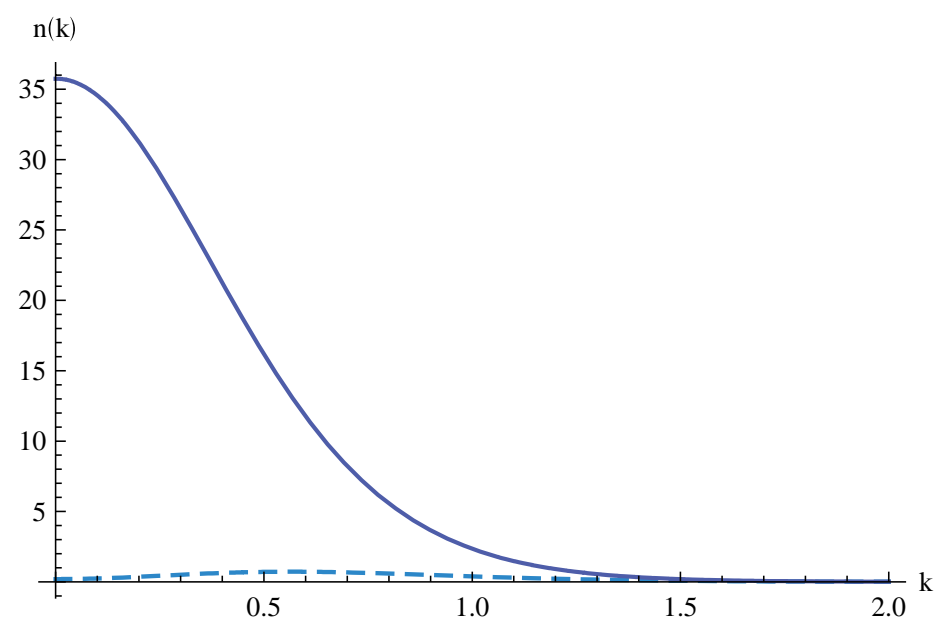

Fig. 1. Occupation numbers for temporal (continuous line) and longitudinal (dashed line) photons in the out region versus $k$ in $\eta_{0}^{-1}$ units.

residual ones. The general solution to the modified equations (5) can be written as

$$
\mathcal{A}_{\mu}=\mathcal{A}_{\mu}^{(1)}+\mathcal{A}_{\mu}^{(2)}+\mathcal{A}_{\mu}^{(s)}+\partial_{\mu} \theta
$$

where $\mathcal{A}_{\mu}^{(i)}$, with $i=1,2$, are the two transverse modes of the massless photon; $\mathcal{A}_{\mu}^{(s)}$ is the new scalar state, which is the mode that would have been eliminated if we had imposed the Lorenz condition; and $\partial_{\mu} \theta$ is a pure gauge mode which can be eliminated. In order to quantize the free theory, we perform the mode expansion for the three physical states:

$$
\mathcal{A}_{\mu}=\int d^{3} \mathbf{k}\left[\sum_{\lambda=1,2, s}\left(\mathbf{a}_{\lambda}(k) \mathcal{A}_{\mu k}^{(\lambda)}+\mathbf{a}_{\lambda}^{\dagger}(k) \overline{\mathcal{A}_{\mu k}^{(\lambda)}}\right)\right] .
$$

In fact, the three modes can be chosen to have positive normalization and, for $\xi=1 / 3$, the canonical commutation relations are satisfied:

$$
\left[\mathbf{a}_{\lambda}(\mathbf{k}), \mathbf{a}_{\lambda^{\prime}}^{\dagger}\left(\mathbf{k}^{\prime}\right)\right]=\delta_{\lambda \lambda^{\prime}} \delta^{(3)}\left(\mathbf{k}-\mathbf{k}^{\prime}\right), \quad \lambda, \lambda^{\prime}=1,2, s,
$$

with a positive sign for the three physical states, i.e. there are no negative norm states in the theory, which in turn guarantees that there are no negative energy states (ghosts). Moreover, as shown in Refs. 5 and 9, the theory does not exhibit either local gravity inconsistencies or classical instabilities.

As shown in (6), $\nabla_{\mu} \mathcal{A}^{\mu}$ evolves as a minimally coupled scalar field. This means that on sub-Hubble scales $(|k \eta| \gg 1)$, the field is suppressed by the universe expansion as $\left|\nabla_{\mu} \mathcal{A}_{k}^{(s) \mu}\right| \propto a^{-1}$. Thus, on small scales, the modified Maxwell equations (5) will be physically indistinguishable from the flat space ones. To summarize, from the previous discussion we see that the theory is consistent even though we have not imposed the Lorenz condition. But, moreover, on super-Hubble scales $(|k \eta| \ll 1)$, 
we find that $\left|\nabla_{\mu} \mathcal{A}_{k}^{(s) \mu}\right|=$ const., which, as shown in Ref. 5, implies that the field contributes as an effective cosmological constant in (4).

In order to determine its value, we will assume that the field is generated during inflation from quantum vacuum fluctuations, in a completely analogous way to cosmological metric perturbations. Thus, in an inflationary de Sitter space-time, it is possible to obtain the corresponding dispersion:

$$
\left\langle 0\left|\left(\nabla_{\mu} \mathcal{A}^{\mu}\right)^{2}\right| 0\right\rangle=\int \frac{d k}{k} P_{A}(k),
$$

where $P_{A}(k)=4 \pi k^{3}\left|\nabla_{\mu} \mathcal{A}_{k}^{(s) \mu}\right|^{2}$. In the super-Hubble limit, we obtain for the power spectrum

$$
P_{A}(k)=\frac{9 H_{I}^{4}}{16 \pi^{2}}
$$

with $H_{I}$ the constant Hubble parameter during inflation. Thus the electromagnetic energy density on cosmological scales is given by $\left\langle 0\left|\rho_{A}\right| 0\right\rangle \sim\left(H_{I}\right)^{4}$. The measured value of the dark energy density then requires that $H_{I} \sim 10^{-3} \mathrm{eV}$, which corresponds to an inflationary scale of $M_{I} \sim 1 \mathrm{TeV}$. Thus we see that the cosmological constant value can be naturally explained in terms of physics at the electroweak scale.

Once the field is produced during inflation, its cosmological evolution can be easily calculated. ${ }^{5}$ We find that in cosmological time $[d t=a(\eta) d \eta]$, the homogeneous temporal component $A_{0}(t)$ (the electric potential) is constant during inflation and

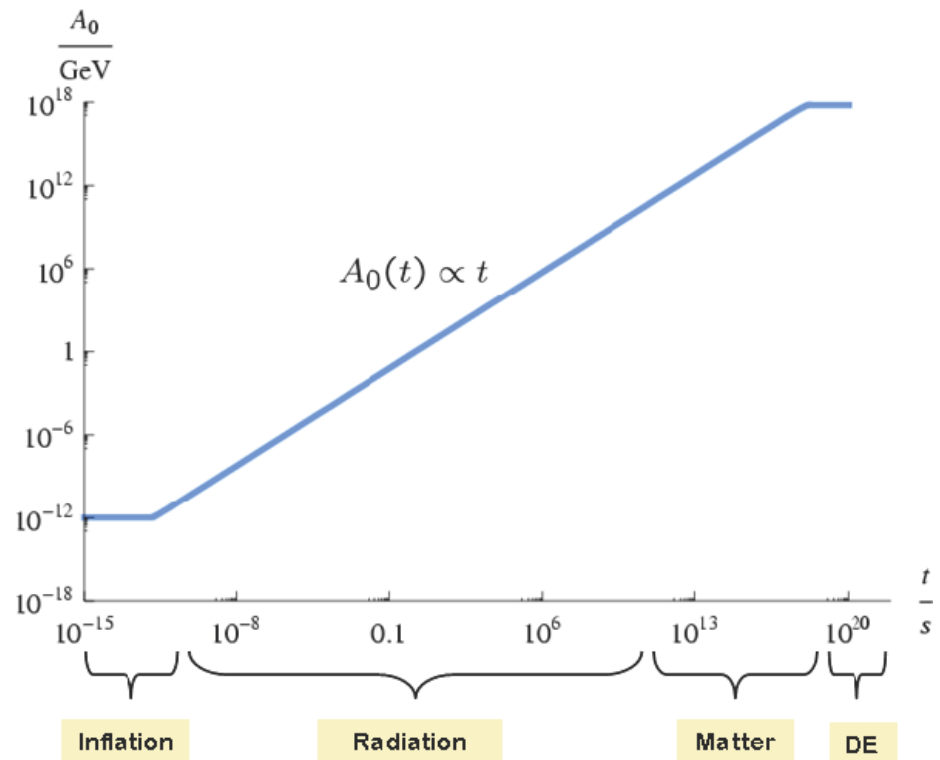

Fig. 2. Cosmological evolution of the electric potential $A_{0}$ from electroweak scale inflation until the present. 
grows as $t$ during matter and radiation eras. When the electromagnetic dark energy starts dominating, $A_{0}(t)$ becomes also constant. The spatial components on superHubble scales $\mathbf{A}(t)$ are shown to grow more slowly than $A_{0}(t)$ and can be neglected. In Fig. 2 we show the cosmological evolution of the electric potential from its initial value generated in inflation $\left[A_{0}\left(t_{I}\right) \sim 10^{-3} \mathrm{eV}\right]$ up to its present value $A_{0}\left(t_{0}\right) \sim$ $0.3 M_{P}$, with $M_{P} \sim 10^{19} \mathrm{GeV}$ the Planck mass.

In conclusion, we have discussed the possibility that the true theory of electromagnetism contains three and not only two physical degrees of freedom. Although the new scalar state is completely decoupled from the conserved currents, it can be gravitationally amplified during inflation, giving rise to the observed dark energy density. The accelerated expansion of the universe would then be the natural consequence of the existence of an absolute electric potential in the universe.

\section{Acknowledgments}

This work has been supported by the Ministerio de Ciencia e Innovación (Spain) projects FIS 2008-01323 and FPA 2008-00592, UCM-Santander PR34/07-15875, CAM/UCM 910309 and MEC grant BES-2006-12059.

\section{References}

1. A. S. Goldhaber and M. M. Nieto, arXiv:0809.1003 (hep-ph).

2. A. L. Maroto, J. Cosmol. Astropart. Phys. 0605 (2006) 015.

3. J. Beltrán Jiménez and A. L. Maroto, Phys. Rev. D 76 (2007) 023003.

4. J. Beltrán Jiménez and A. L. Maroto, arXiv:0903.4672 (astro-ph.CO).

5. J. Beltrán Jiménez and A. L. Maroto, J. Cosmol. Astropart. Phys. 0903 (2009) 016 [arXiv:0811.0566 (astro-ph)].

6. C. Itzykson and J. B. Zuber, Quantum Field Theory (McGraw-Hill, 1980).

7. N. N. Bogoliubov and D. V. Shirkov, Introduction to the Theory of Quantized Fields (Interscience, 1959).

8. N. D. Birrell and P. C. W. Davies, Quantum Fields in Curved Space (Cambridge, 1982).

9. J. Beltrán Jiménez and A. L. Maroto, J. Cosmol. Astropart. Phys. 0902 (2009) 025 [arXiv:0811.0784 (astro-ph), arXiv:0812.1970 (astro-ph)]. 
Copyright of International Journal of Modern Physics D: Gravitation, Astrophysics \& Cosmology is the property of World Scientific Publishing Company and its content may not be copied or emailed to multiple sites or posted to a listserv without the copyright holder's express written permission. However, users may print, download, or email articles for individual use. 